\title{
UV/VIS spectral properties of novel natural products from Turkish lichens
}

\author{
Yevgen Posokhov, ${ }^{1,2}$ Şule Erten, ${ }^{1,3}$ Ömer Koz, ${ }^{4}$ H. Anıl, ${ }^{4}$ \\ Süheyla Kırmızıgül, ${ }^{4}$ and Sıddık Içli ${ }^{1}$ \\ ${ }^{1}$ Solar Energy Institute, Ege University, Bornova, 35100 Izmir, Turkey \\ ${ }^{2}$ Institute for Chemistry at Kharkov National University, 61077 Kharkov, Ukraine \\ ${ }^{3}$ Dokuz Eylul University, Faculty of Science Edu., Depatrment of Chemistry, Buca, Izmir, Turkey \\ ${ }^{4}$ Ege University, Faculty of Science, Depatrment of Chemistry, Bornova, Izmir, Turkey
}

\begin{abstract}
UV/VIS spectral characteristics of three new biologically active natural products, isolated from Turkish lichens, have been investigated in solvents of various polarity and proton donating ability. The effect of the solvent on spectral characteristics has been estimated. Quantum chemical calculations with the optimization of molecular geometry were done with the full-valent semiempirical methods AM1 and PM3 for conformational analysis and in order to discuss the charge distributions and dipole moments in the ground and in the excited states.
\end{abstract}

\section{INTRODUCTION}

Lichens have in great variety metabolic products, some of which appear to occur naturally only in lichens and others are also present in higher plants and fungi. Some known secondary metabolites of lichens are depsides, depsidones, benzoxazine, benzofurane, usnic acid and antraquinone derivatives [1-6]. Because of their metabolites, lichens are widely used as commercially in perfume dye and drug industry [7-11]: about 60 lichen species are present in some different types of commercial drugs such as antimicrobial, anticancer, antiallergen, immunugical and expectoral [12-15].

The practical usage in pharmaceutical industry arises the importance on studies of physico-chemical and spectral properties of the isolated compounds from lichens, e.g., the knowledge on the spectral properties of isolated compounds could facilitate their biochemical analysis [16].

We already reported the molecular structure justification of the three compounds $(\mathbf{1}, \mathbf{2}, \mathbf{3})$ isolated from Turkish lichens Pseudevernia furfuracea, Evernia prunastri, Letharia vulpina, respectively, [17].

In this report we wish to present the results of our studies on UV/VIS spectral properties of the abovementioned compounds $\mathbf{1}, 2,3$.<smiles>COc1cc(C)c(C(=O)O)c(O)c1C</smiles><smiles>COc1cc(O)cc(C)c1C(=O)Oc1ccc(N)c(C(C)=O)c1Cl</smiles><smiles>COc1ccc(/C(C(=O)O)=C2/C=C(c3ccccc3)C(=O)O2)cc1</smiles>

3

\section{EXPERIMENTAL DETAILS}

2.1. Materials. The separation, identification and purification of compounds 1, 2, 3 were described earlier [17]. The organic solvents used were all of spectrophotometric grade and were used as supplied from Fluka.

2.2. Spectroscopic measurements. The electronic absorption spectra were measured using Jasco V-530 UV/VIS spectrophotometer.

2.3. Theoretical calculations. Semi-empirical calculations were performed using the original AM1 [18], PM3 [19], ZINDO/S [20] parametrisations (included in HYPER Chem package). Restricted Hartree-Fock (RHF) formalism was used.

AM1 method is known to have better parameterization in comparison with PM3 for geometry optimization, though, intramolecular hydrogen-bonding is 
better parameterized for PM3. ZINDO/S was used for calculations of the UV/VIS spectra because this method was specially parameterized for electronic spectroscopy.

The calculations were carried out with full ground state geometry optimization without any assumption of symmetry: Polak-Ribiere (conjugate gradient) geometry optimization algorithm was used with convergence cut-off criterion $0.1 \mathrm{kcal} / \mathrm{mol}$.

Excited state calculations were conducted by means of single point calculations (closed shell, singles) of the structures with already optimized ground state geometry: CI matrix with 3 HOMO's and 3 LUMO's has been used.

Mulliken charges [21] were used to discuss the dipolar moments.

\section{RESULTS AND DISCUSSIONS}

3.1. Absorption properties. In Tables 1, 2, 3 the absorption wavelengths of the compounds 1, 2, 3 are presented. Figures 1, 2, 3 show the absorption spectra of 1, 2, 3 in various solvents with different polarity and proton donating ability.

Absorption spectra of 1, 2, 3 in the region 200$450 \mathrm{~nm}$ consist of four bands of $\pi-\pi^{*}$ nature, the $\mathrm{n}-\pi^{*}$ absorption bands are hidden by the more intense longwavelength $\pi-\pi^{*}$ bands.

The absorption spectra of compound $\mathbf{1}$ could be considered as the spectra of substituted orthohydroxybenzoic acid. Such chromophoric fragments could be distinguished in 1: benzoic acid $\left(\lambda^{1} \max \right.$ $\left.227 \mathrm{~nm}, \log \varepsilon 4.15 ; \lambda^{2} \max 267 \mathrm{~nm}, \log \varepsilon 3.25\right)$, phenol $\left(\lambda^{1}{ }_{\max } 215 \mathrm{~nm}, \log \varepsilon 4.0 ; \lambda^{2} \max 273 \mathrm{~nm}, \log \varepsilon 3.25\right)$, toluene $\left(\lambda_{\max } 262 \mathrm{~nm}, \log \varepsilon 2.48\right)$ [22]. The donor substituents shifted the spectra of the above chromophoric fragments to long-wavelength region. Molecular extinction coefficients for compound 1 (see inscription under Table 1) are much grater than those for the reference compounds (benzoic acid, phenol), i.e., hyperchromic effect is observed with the introduction of donor substituents into phenyl ring of benzoic acid.

The absorption spectra of $\mathbf{2}$ are additive and should be considered in the light of the spectra of the hydroxybenzoic acids and their derivatives. Substituents clearly exert bathochromic effects [22].

The absorption spectra of compound 3 originated from the presence of the two chromophoric fragments: 1,4-diphenyl-trans-trans-butadiene-1, 3 and trans, trans-muconic acid [22].

Intensive conformational search was conducted for $\mathbf{1}, \mathbf{2}, \mathbf{3}$. We started the calculations from different starting geometries and, for each compound, the geometry search converged to one of the two most stable conformers, designated as $\boldsymbol{I}$ and $\boldsymbol{I I}$ (see Figures 4, 5, 6; Table 4). Other possible conformers were neglected, because the rotation of the peripheral methyl groups could not affect the absorption spectra considerably.

The conformers $\boldsymbol{I}$ have greater ground state dipole moment values, calculated with AM1 and PM3 parameters, than corresponding values for conformers II (see Table 4), calculated with the same parameters. Thus, with growth of solvent polarity, the conformers $I$ will be more favorable in comparison with the corresponding conformers $\boldsymbol{I} \boldsymbol{I}$.

Quantum-chemical calculations of UV/VIS spectra for compounds 1, 2, 3 in vacuum were made with the use of AM1, PM3, ZINDO/S parameters. The results of the calculations are presented in Table 5.

In order to check the validity of $3 \times 3 \mathrm{CI}$ matrix for excited state calculations, a few calculations with CI matrix $5 \times 5$ were made, but no considerable difference in results of both calculations was noticed. For this reason, the majority of the excited state calculations was performed with the usage of $3 \times 3 \mathrm{CI}$ matrix.

The comparison of the calculated spectra with the experimental ones shows that the best coincidence of the experimental and calculated UV/VIS data is observed when ZINDO/S parameters are used in order to calculate spectra of 1, when AM1 and PM3 parameters are used in order to calculate spectra of 2 , and, when AM1 and ZINDO/S parameters are used in order to calculate spectra of compound 3.

In general, the tendencies in calculated oscillator strength ratio's for calculated absorption bands of $\mathbf{1}$, 2, 3 (Table 5) qualitatively agree with the corresponding ratio's of molar extinction coefficients for $\lambda_{1}, \lambda_{2}$, $\lambda_{3}$ bands of 1, 2, 3 (see inscriptions under Tables 1, 2, 3 ). The only exception is observed for long-wavelength bands of compound 3: the calculated oscillator strength ratio for $\lambda_{1} \sim 370 \mathrm{~nm}$ and $\lambda_{2} \sim 286 \mathrm{~nm}$ is not in accordance with the corresponding ratio of molar extinction coefficients (see inscription under Table 3).

According to the calculations of UV/VIS spectra of 1, 2, 3 in vacuum (Table 5), the long-wavelength absorption bands of the conformers $\boldsymbol{I}$ are shifted to higher energies in comparison with the corresponding absorption bands of the conformer $\boldsymbol{I I}$.

By the growth of solvent polarity, short-wavelength shifts in the absorption maxima are observed (up to $5,6,10 \mathrm{~nm}$ for $1,2,3$, respectively, see Tables $1,2,3$ and Figures 1, 2, 3). This fact could be caused by such possibilities: (a) probably, in polar solvents the energies of the conformers $\boldsymbol{I}$ and $\boldsymbol{I I}$, calculated for 1, 2, 3 in vacuum (Table 4), will change because of solvatation, and, perhaps, the energy difference between $\boldsymbol{I}$ and $\boldsymbol{I I}$ will increase because of the greater solvent stabilization of the more polar conformers $I$, hence, conformational equilibria between $\boldsymbol{I}$ and $\boldsymbol{I I}$ will be shifted in the direction of $\boldsymbol{I}$ for which, as shown by the calculations (AM1, PM3, ZINDO/S; see Table 5), the positions of the long-wavelength maxima are slightly shifted to higher energies; (b) the dipole moments of the some conform- 
Table 1. UV/VIS spectroscopic data* of compound 1 in solvents with different polarities and hydrogen bonding abilities.

\begin{tabular}{|c|c|c|c|c|c|c|}
\hline Solvent & $\varepsilon$ & $n$ & $\lambda^{1}$ abs & $\lambda^{2} a b s$ & $\lambda^{3} \mathrm{abs}$ & $\lambda^{4}{ }_{a b s}$ \\
\hline Tetrachloromethane & 2.24 & 1.4574 & 307 & 266 & - & - \\
\hline Benzene & 2.28 & 1.5011 & 306 & - & - & - \\
\hline Toluene & 2.38 & 1.4961 & 306 & - & - & - \\
\hline Chloroform & 4.70 & 1.4459 & 305 & 266 & - & - \\
\hline Ethyl acetate & 6.02 & 1.3723 & 304 & 267 & - & - \\
\hline Tetrahydrofuran & 7.6 & 1.4076 & 304 & 266 & 237 & 214 \\
\hline Dichloromethane** & 8.90 & 1.4242 & 303 & 265 & 227 & - \\
\hline Iso-propanol & 18.3 & 1.3747 & 303 & 266 & 226 & - \\
\hline Methanol & 32.63 & 1.3286 & 303 & 268 & 219 & - \\
\hline Acetonitrile*** & 36.2 & 1.3441 & 302 & 268 & 217 & - \\
\hline Dimethylformamide & 36.7 & 1.4303 & 302 & 268 & - & - \\
\hline
\end{tabular}

*Here $\varepsilon$ and $n$-dielectric permeability and refractive index of the solvent; $\lambda^{1-4}$ abs - are the positions of the maxima in the absorption spectra $(\mathrm{nm})$.

** Molecular extinction coefficients were calculated to be: $\varepsilon_{1}\left(\lambda^{1}{ }_{\text {abs }}\right)=9300 ; \varepsilon_{2}\left(\lambda^{2}{ }_{\text {abs }}\right)=28500 ; \varepsilon_{3}\left(\lambda^{3}\right.$ abs $)=31700$;

*** Molecular extinction coefficients were calculated to be: $\varepsilon_{1}\left(\lambda^{1}{ }_{\text {abs }}\right)=9500 ; \varepsilon_{2}\left(\lambda^{2}{ }_{\text {abs }}\right)=30500 ; \varepsilon_{3}\left(\lambda^{3}\right.$ abs $)=104700$.

Table 2. UV/VIS spectroscopic data* of compound 2 in solvents with different polarities and hydrogen bonding abilities.

\begin{tabular}{|c|c|c|c|c|c|}
\hline Solvent & $\varepsilon$ & $n$ & $\lambda^{1}{ }_{a b s}$ & $\lambda^{2} a b s$ & $\lambda^{3}$ abs \\
\hline Tetrachloromethane & 2.24 & 1.4574 & 312 & - & - \\
\hline Benzene & 2.28 & 1.5011 & 312 & - & - \\
\hline Toluene & 2.38 & 1.4961 & 311 & - & - \\
\hline Chloroform & 4.70 & 1.4459 & 310 & 270 & 242 \\
\hline Ethyl acetate & 6.02 & 1.3723 & 309 & 268 & - \\
\hline Tetrahydrofuran & 7.6 & 1.4076 & 309 & - & - \\
\hline Dichloromethane $* *$ & 8.90 & 1.4242 & 309 & 270 & 237 \\
\hline Iso-propanol & 18.3 & 1.3747 & 303 & 268 & 214 \\
\hline Methanol & 32.63 & 1.3286 & 303 & 268 & 209 \\
\hline Acetonitrile*** & 36.2 & 1.3441 & 306 & 270 & 214 \\
\hline Dimethylformamide & 36.7 & 1.4303 & 306 & 268 & - \\
\hline
\end{tabular}

*Here $\varepsilon$ and $n$-dielectric permeability and refractive index of the solvent; $\lambda^{1-3}$ abs - are the positions of the maxima in the absorption spectra $(\mathrm{nm})$.

${ }^{* *}$ Molecular extinction coefficients were calculated to be: $\varepsilon_{1}\left(\lambda^{1}{ }_{\text {abs }}\right)=15600 ; \varepsilon_{2}\left(\lambda^{2}\right.$ abs $)=32400 ; \varepsilon_{3}\left(\lambda^{3}{ }_{\text {abs }}\right)=330500$;

*** Molecular extinction coefficients were calculated to be: $\varepsilon_{1}\left(\lambda^{1}{ }_{\text {abs }}\right)=16700 ; \varepsilon_{2}\left(\lambda^{2}{ }_{\text {abs }}\right)=29100 ; \varepsilon_{3}\left(\lambda^{3}{ }_{\text {abs }}\right)=230100$.

Table 3. UV/VIS spectroscopic data* of compound 3 in solvents with different polarities and hydrogen bonding abilities.

\begin{tabular}{lcccccc}
\hline Solvent & $\varepsilon$ & $n$ & $\lambda^{1}$ abs & $\lambda^{2}{ }_{\text {abs }}$ & $\lambda^{3}$ abs & - \\
\hline Tetrachloromethane & 2.24 & 1.4574 & 379 & 278 & - & - \\
Benzene & 2.28 & 1.5011 & 379 & - & - & - \\
Toluene & 2.38 & 1.4961 & 378 & - & - & - \\
Chloroform & 4.70 & 1.4459 & 377 & 275 & 249 & - \\
Ethyl acetate & 6.02 & 1.3723 & 376 & 275 & 275 & 237 \\
Dichloromethane** & 8.90 & 1.4242 & 374 & 279 & 235 & 206 \\
Iso-propanol & 18.3 & 1.3747 & 371 & 277 & 232 & 207 \\
Methanol & 32.63 & 1.3286 & 370 & 279 & 238 & 209 \\
Acetonitrile*** & 36.2 & 1.3441 & 369 & & - \\
\hline
\end{tabular}

${ }^{*}$ Here $\varepsilon$ and $n$-dielectric permeability and refractive index of the solvent; $\lambda^{1-4}$ abs - are the positions of the maxima in the absorption spectra (nm).

** Molecular extinction coefficients were calculated to be: $\varepsilon_{1}\left(\lambda^{1}{ }_{\text {abs }}\right)=21900 ; \varepsilon_{2}\left(\lambda^{2}{ }_{\text {abs }}\right)=96120 ; \varepsilon_{3}\left(\lambda^{3}\right.$ abs $)=24100$;

*** Molecular extinction coefficients were calculated to be: $\varepsilon_{1}\left(\lambda^{1}{ }_{\text {abs }}\right)=32100 ; \varepsilon_{2}\left(\lambda^{2}{ }_{\text {abs }}\right)=34900 ; \varepsilon_{3}\left(\lambda^{3}\right.$ abs $)=36200$; $\varepsilon_{4}\left(\lambda_{\text {abs }}^{4}\right)=38900$. 


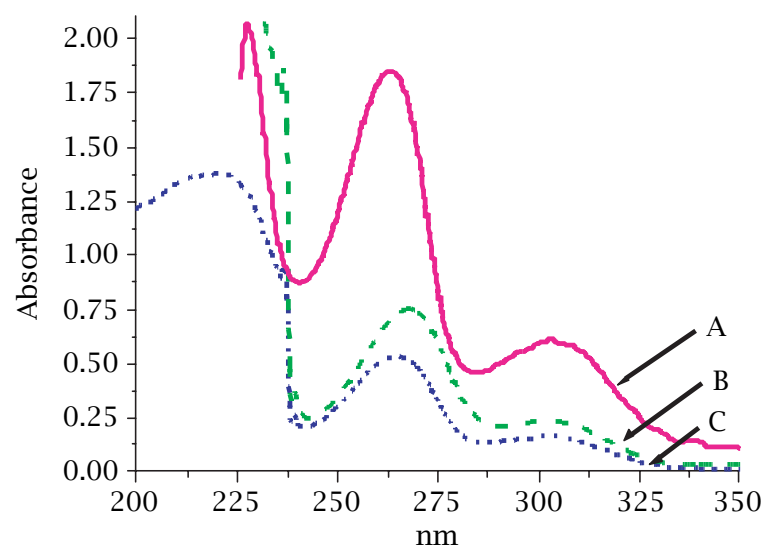

Figure 1. Absorption spectra of compound 1: A- in dichloromethane, B- in acetonitrile, C- in iso-propanol.

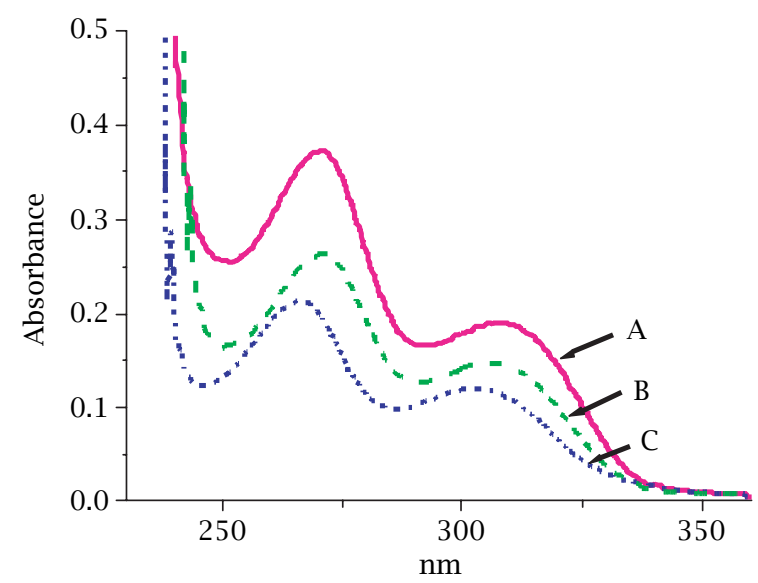

Figure 2. Absorption spectra of compound 2: A- in dichloromethane, B- in acetonitrile, C- in iso-propanol.

ers of 1, 2, 3 in excited state is lower than the corresponding dipole moments in ground state. Increasing solvent polarity stabilizes the ground state to a greater degree than the electronically excited state and, the absorption spectrum tends to shift to shorter wavelength with the increasing solvent polarity [23].

In order to elucidate whether the latter possibility occurs, quantum-chemical calculations with AM1 [18] and with PM3 [19] parameters were used to estimate the excited state dipole moments of comp. 1, 2, 3 (see Table 4).

As could be seen from the Table 4 , the values of the excited state dipole moment $\mu_{e}$ for conformer $\boldsymbol{I I}$ of compound 1 (as calculated with PM3), for conformer I of compound 2 (as calculated with AM1 and PM3) and for conformer II of compound $\mathbf{3}$ (as calculated with PM3), are greater than the corresponding values of ground state dipole moment $\mu_{g}$, calculated with the

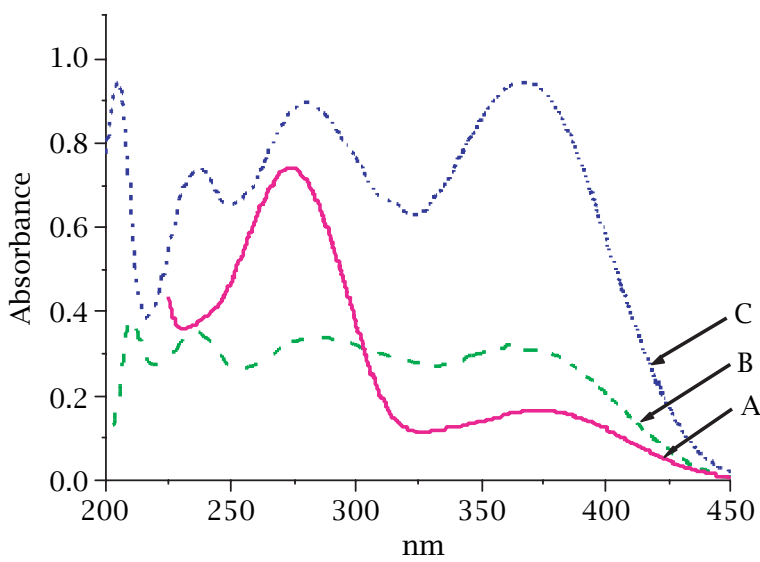

Figure 3. Absorption spectra of compound 3: A- in dichloromethane, $\mathrm{B}$ - in acetonitrile, $\mathrm{C}$ - in iso-propanol.

use of the same parameters. In general, the changes of the dipole moments on excitation are not considerable.

In contrast to solvent polarity, which affects the position of the long-wavelenth absorption band of $\mathbf{1}$, according to the data presented in Table 1, solvent H-bond donating and H-bond accepting ability have practically no influence on the position of the longwavelength maximum in absorption spectra of comp. 1.

The blue (short-wavelength) shifts of longwavelength absorption maxima are observed in proton-donating solvents, such as iso-propanol or methanol, for compound 2 (see Table 2). According to the quantum chemical calculations (see Figure 5) electronic density redistributes from amino group to carbonyl group on excitation. Taking into account this fact, one could explain that the blue shifts of long-wavelength absorption maxima of compound 2 in proton donating solvents caused by the interaction of hydrogen-bond donor solvents with unshared valence electron pairs of the amino group. The latter is charge donor in the excited state. This interaction prevents the charge transfer from the amino group to carbonyl group in an excited state, and, consequently destabilizes the charge-transfer excited state relative to the ground state, so that the absorption spectra tend to shift to higher energies with increasing hydrogen-bond donor capacity of the solvent [23].

According to the quantum chemical calculations for compound 3 (see Figure 6), electronic density redistributes to lactone carbonyl group on excitation. Taking into account this fact, one could expect longwavelength shift of absorption maxima of compound 3 in proton donating iso-propanol and methanol caused by the interaction of hydrogen-bond donor solvents with unshared valence electron pairs of the lactone carbonyl group, which is charge acceptor in the excited state. 


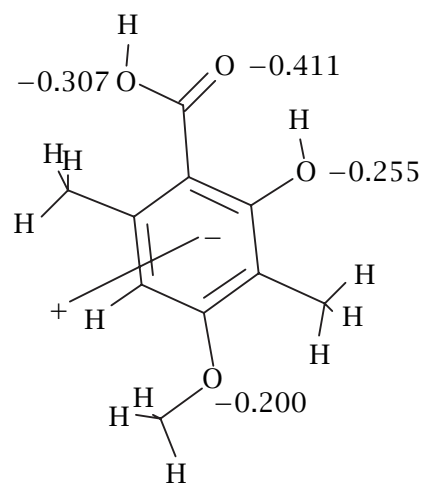

$I$

(a) Ground State

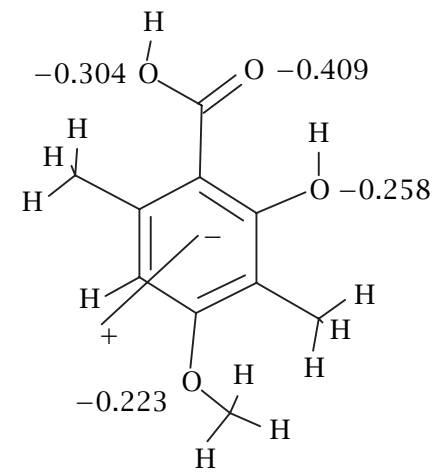

II

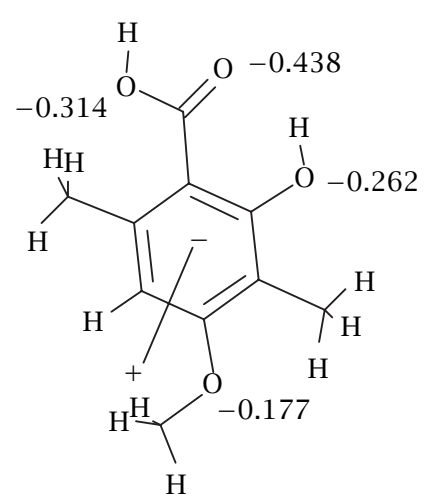

I

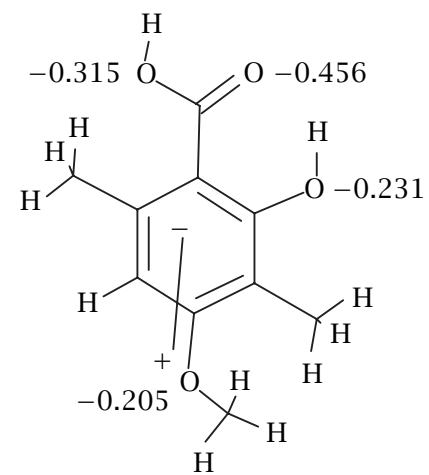

II

(b) Excited State

Figure 4. Calculated by AM1 method charge distribution and dipole moment directions in $S_{0}$ (a) and in $S_{1}$ (b) electronic state of the most stable conformers (I and II) of compound $\mathbf{1}$ in vacuum.

Nevertheless, no spectral shifts of the longwavelength band of compound 3 are observed in polar proton-donating iso-propanol and methanol in comparison with polar aprotic acetonitrile (see Table 3).

Probably, the expected long-wavelength shift is compensated by the short-wavelength shift of absorption maxima, the latter is caused by the interaction of hydrogen-bond donor solvents with unshared valence electron pairs of methoxy group, which is charge donor in the excited state.

It should be noted that absorption spectrum of comp. 3 in dichloromethane solution differs from the absorption spectra of the same compound in the other solvents used: i.e., the ratio of intensities of the bands $\lambda_{1}$ and $\lambda_{2}$ (see Figure 3, Table 3) is different from the corresponding ratios for the other solvents used. Such anomalous behavior of comp. 3 in dichloromethane solution will be the subject for the future study.

Fluorescence is known to be a powerful tool for pharmaco-chemical analysis, provided that analyte is fluorescent [23].
Unfortunately, all the studied compounds are nonfluorescent in all the solvents used (the roughly estimated quantum yields are very low $\sim 10^{-3}$ ).

For comp. 1, provided that the presence of methyl substituents in phenyl ring do not suppress the hydroxy group acidity on excitation, the fluorescence quenching could be linked with the excited state intramolecular proton transfer (ESIPT) of the 2-hydroxy proton to the carbonyl oxygen followed by radiationless decay and fast back proton transfer [24].

Otherwise, if the presence of methyl substituents in phenyl ring of $\mathbf{1}$ suppress the hydroxy group acidity on excitation, and, the ESIPT does not occur, efficient intersystem crossing (with the participation of singlet or triplet states of $n \pi^{*}$ nature, introduced into the system by the presence of the carbonyl group) could be considered as the possible cause of the fluorescence quenching of $\mathbf{1}$.

Few cases of the ESIPT with amino group as protonodonor are reported in literature [24, 25], though, one of such cases is the ESIPT in 2-amino-3-naphtoic acid 


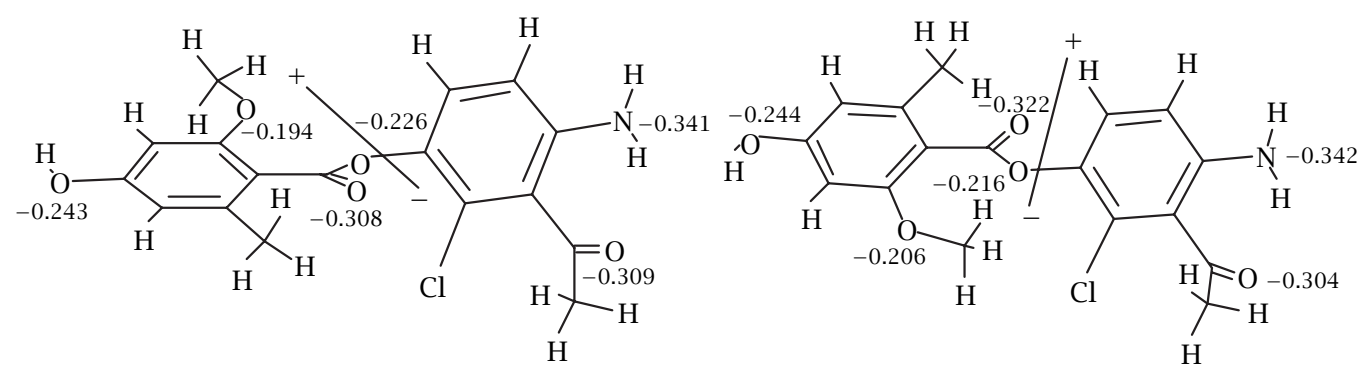

I

II

(a) Ground State

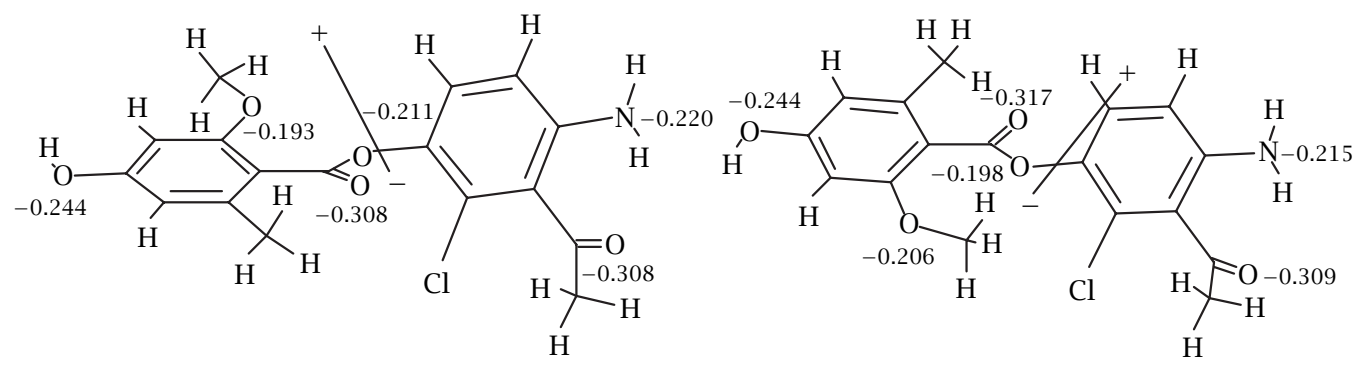

I

II

(b) Excited State

Figure 5. Calculated by AM1 method charge distribution and dipole moment directions in $S_{0}$ (a) and in $S_{1}$ (b) electronic state of the most stable conformers (I and II) of compound 2 in vacuum.

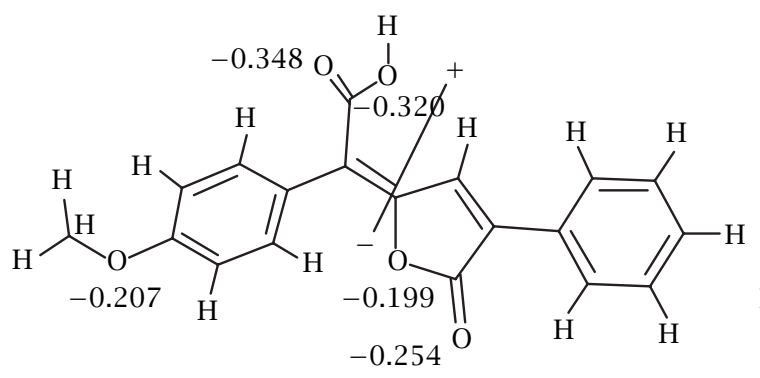

I

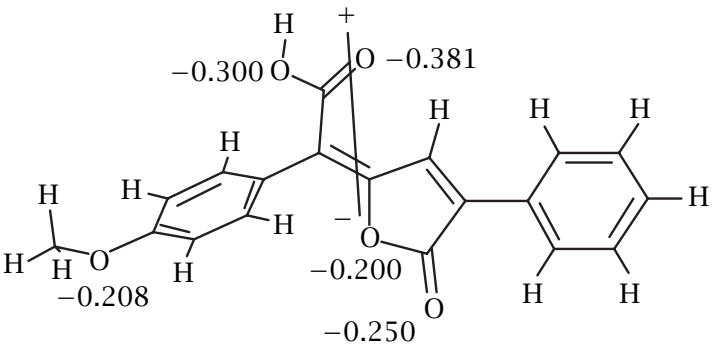

II

(a) Ground State

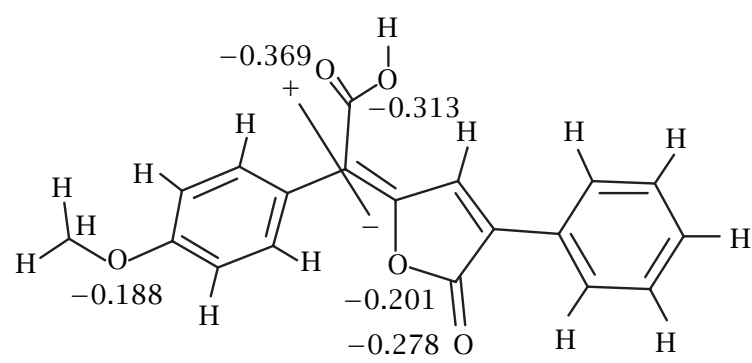

I

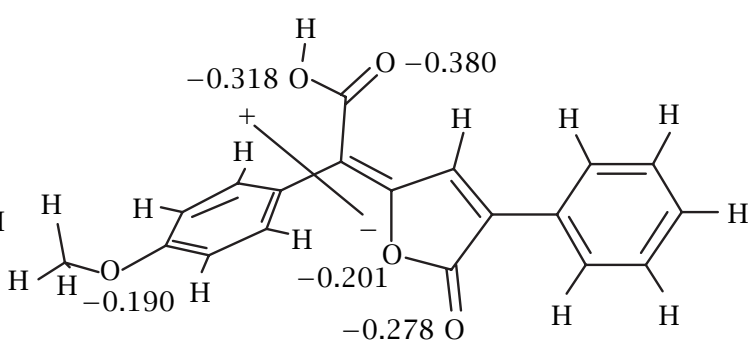

II

(b) Excited State

Figure 6. Calculated by AM1 method charge distribution and dipole moment directions in $S_{0}$ (a) and in $S_{1}$ (b) electronic state of the most stable conformers (I and II) of compound 3 in vacuum. 
Table 4. Relative heats of formation* $\left(H_{f}, \mathrm{kcal} / \mathrm{mol}\right)$, total energy ${ }^{* * *}\left(E_{T}, \mathrm{kcal} / \mathrm{mol}\right)$ and dipole moments $\left(\mu\right.$, Debye) for $S_{0}$ and $S_{1}$ states of the most stable conformers of 1, 2, 3 in vacuum calculated with AM1 and PM3 parameters.

\begin{tabular}{|c|c|c|c|c|c|c|c|c|c|c|c|}
\hline \multirow{3}{*}{ Comp. } & \multirow{3}{*}{ Conformer } & \multicolumn{5}{|c|}{ AM1 } & \multicolumn{5}{|c|}{ PM3 } \\
\hline & & \multicolumn{3}{|c|}{$\mathrm{S}_{0}$} & \multicolumn{2}{|c|}{$S_{1}$} & \multicolumn{3}{|c|}{$\mathrm{S}_{0}$} & \multicolumn{2}{|c|}{$S_{1}$} \\
\hline & & $H_{f}$ & $E_{T}$ & $\mu_{g}$ & $H_{f}$ & $\mu_{e}$ & $H_{f}$ & $E_{T}$ & $\mu_{g}$ & $H_{f}$ & $\mu_{e}$ \\
\hline \multirow{2}{*}{1} & $I$ & 0 & -62909.0 & 3.68 & 83.0 & 3.91 & 4.94 & -58689.3 & 3.43 & 87.69 & 3.80 \\
\hline & II & 2.76 & -62906.2 & 1.80 & 85.28 & 2.36 & 0 & -58694.3 & 2.42 & 82.45 & 2.11 \\
\hline \multirow{2}{*}{2} & $I$ & 0 & -105593.3 & 4.25 & 94.87 & 3.70 & 0 & -97033.1 & 4.31 & 102.0 & 3.77 \\
\hline & II & 1.52 & -105591.7 & 0.85 & 94.06 & 1.13 & 3.57 & -97029.5 & 0.55 & 96.58 & 1.75 \\
\hline \multirow{2}{*}{3} & $I$ & 0 & -97438.3 & 3.21 & 81.02 & 3.19 & 0 & -90705.2 & 2.83 & 87.32 & 3.01 \\
\hline & II & 0.31 & -97438.0 & 2.91 & 80.91 & 3.86 & 0.15 & -90705.1 & 2.80 & 91.69 & 2.54 \\
\hline
\end{tabular}

*Here, heat of formation for the most stable conformer for each compound was taken as zero.

** Presented only for $\mathrm{S}_{0}$ states of the conformers $\boldsymbol{I}$ and $\boldsymbol{I I}$.

and its methyl ester [25]. Taking into account this fact, one could suggest that fluorescence quenching of $\mathbf{2}$ is caused by the excited state proton transfer of 2-amino proton to the carbonyl group followed by efficient nonradiative deactivation in phototautomer form.

On the other hand, another cause of the observed fluorescence quenching of 2 could be efficient intersystem crossing.

In compound 3 there is no possibility for the ESIPT, but another two possibilities of fluorescence quenching occur: (i) intersystem crossing; (ii) fast rotation around double bond to a perpendicular minimum of the $S_{1}$ state followed by surface crossing to $S_{0}$ surface. The second possibility seems less probable: fast rotation around double bond is usually linked with ethylenelike cis-trans photoisomerisation [26], but, according to experimental results for compound 3, no spectral changes were noticed after a long-time irradiation with the use of UV/VIS lamp (the light source of Jasco spectrophotometer).

Thus, the possibility of the effective quenching processes with the participation of singlet or triplet states of $n-\pi^{*}$ nature, introduced into the molecules $1,2,3$ by the presence of the carbonyl groups, could exist for all the studied compounds.

If the lowest-lying transitions of organic substance are of $\pi-\pi^{*}$ type such a substance usually have relatively high fluorescence quantum yield. When a heteroatom is involved in the $\pi$-system an $n-\pi^{*}$ transition may be the lowest-lying transition. This opens the possibility for radiotionless deactivations of the lowest singlet excited state via the efficient intersystem crossing (ISC) of S(n $\left.\pi^{*}\right)$-T $\left(\pi \pi^{*}\right)$ or S( $\left.\pi \pi^{*}\right)$-T(n $\left.\pi^{*}\right)$ type. According to El-Sayed rule [27], the rate of ISC between the singlet and triplet states of the different orbital nature exceeds that for the states of the identical orbital nature up to several orders of magnitude. Owing to this circumstance, the ISC between $\mathrm{n} \pi^{*}$ and $\pi \pi^{*}$ states successfully compete with the radiative depopulation of $\mathrm{S}_{1} *$ energetic level.
This explains the low fluorescence quantum yields of many molecules in which the lowest excited state is $n \pi^{*}$ in nature. This is the case for most of the azo compounds and some compounds containing carbonyl groups and nitrogen heterocycles (with pyridine-type nitrogens) [28].

Many aromatic aldehydes and ketones (e.g., benzophenone. anthrone. 1- and 2-naphthaldehyde) have a low-lying $n \pi^{*}$ excited state and thus exhibit low fluorescence quantum yields, as explained above. The dominant de-excitation pathway is intersystem crossing (whose efficiency has been found to be close to 1 for benzophenone) [29].

According to the absorption spectra of 1, 2, 3 (see Figures 1, 2, 3 and Tables 1, 2, 3), the $\mathrm{S}\left(\mathrm{n} \pi^{*}\right.$ ) levels of $1,2,3$ are found to be higher in energy then corresponding $\mathrm{S}\left(\pi \pi^{*}\right)$ levels (n $\pi^{*}$ absorption bands of $\mathbf{1 ,}, 2$, 3 are hidden by the more intense long-wavelength $\pi \pi^{*}$ bands). Thus, probably, the quenching of the fluorescence for $1,2,3$ is caused by $\mathrm{S}\left(\pi \pi^{*}\right)$-T(n $\left.\pi^{*}\right)$ intersys tem crossing. Though, to prove this, additional future study appears to be necessary.

As it was mentioned above, in cases where the lowest excited states of carbonyl-containing compounds are of the $n \pi^{*}$ type (usually, such cases are observed in non-polar/ non-hydrogen bonding solvents), no fluorescence takes place. However, if $\pi \pi^{*}$ states of carbonyl-containing compounds lies only slightly higher than $n \pi^{*}$ states, the increase of solvent polarity or hydrogen-bonding ability could change the arrangement of the $n \pi^{*}$ and $\pi \pi^{*}$ levels and, hence, rather intense fluorescence can be observed in polar or in protic solvents.

The solvent may not only change the arrangement of the singlet $n \pi^{*}$ and $\pi \pi^{*}$ levels, but also promotes the vibrational spin-orbital interaction between the lowest $\mathrm{S}\left(\pi \pi^{*}\right)$ and the higher $\mathrm{S}\left(\mathrm{n} \pi^{*}\right)$ level with subsequent transition to the triplet level. This vibrational interaction is more probable in hydrocarbon solvents. In polar solvents, such interaction is insignificant, and, as 


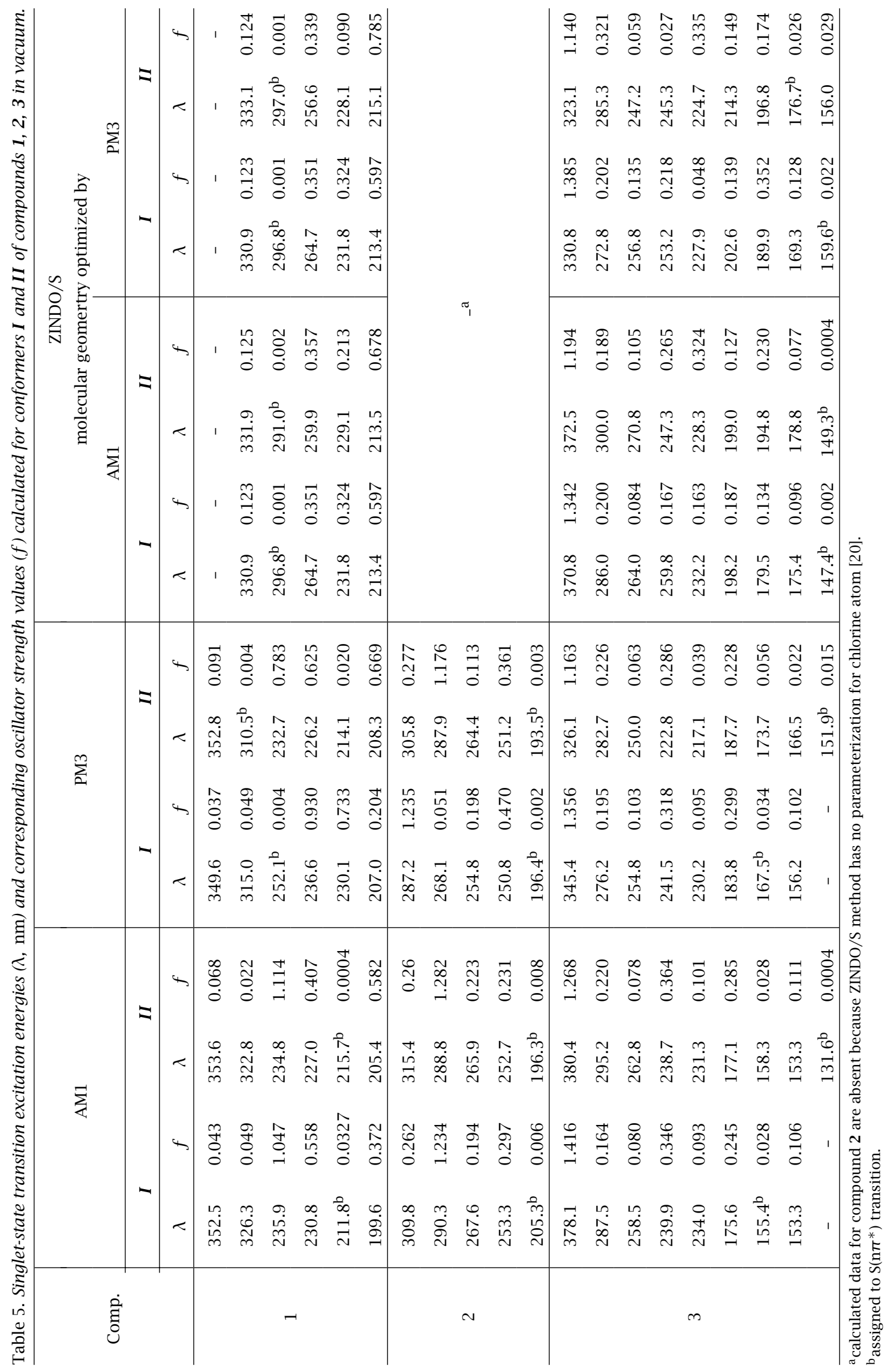


a result-the fluorescence intensity is higher [30]. This fact is considered as the another reason for the increase of fluorescence quantum yield of the compounds, containing $\mathrm{n} \pi^{*}$-levels, with increase of solvent polarity [30].

Nevertheless, unfortunately, in case of compounds 1, 2, 3 the fluorescence emission remains very low even in polar solvents (dichloromethane, acetonitrile).

\section{CONCLUSION}

The UV/VIS spectral properties of the compounds 1, 2, 3, isolated from Turkish lichens Pseudevernia furfuracea, Evernia prunastri, Letharia vulpina, respectively, have been conducted. It has been found that the absorption spectra of all the three compounds shift hypsochromically with the growth of the solvent polarity.

UV/VIS spectral results can be enlightening for monitoring of the above compounds at pharmacochemical analysis.

\section{ACKNOWLEDGMENTS}

Ph.D. student Özgül Hakli is acknowledged for the measurements of fluorescence spectra. The authors express their gratitude to Alexander von Humboldt Foundation of Germany, Ege University Research Funds Office and TUBITAK-Scientific and Technical Research Council of Turkey for their support.

\section{REFERENCES}

[1] Y. Asahina and S. Shibata, Chemistry of Lichen Substances, Asher, A, Co. Ltd. Vaals - Amsterdam, 1971.

[2] C. F. Culberson, Chemical and Botanical Guide to Lichen Products, The University of North Carolina Press, Chabel Hill, 1969.

[3] C. F. Culberson and J. A. Elix, Lichen Substances: Methods in Plant Biochemistry (P. M. Dey and J. B. Harborne, eds.), vol. 1, Academic Press, London, 1989.

[4] J. A. Elix and D. A. Venables, Mycotaxon 47 (1993), 275.

[5] S. Huneck and I. Yoshimura, Identification of Lichen Substances, Springer, 1966.

[6] U. Zeybek, H. T. Lumbsch, G. B. Feige, J. A. Elix, and V. John, Crypt. Bot. 3 (1993), 263.
[7] I. K. Bergwein, Dragoca Rep. 3 (1972), 48.

[8] K. Karamanoğlu, Türk Biol. Der. 2 (1966), 114.

[9] T. H. Moxham, Dragoco Rep. 2 (1981), 31.

[10] T. H. Moxham, The Commercial Exploitation of Lichens for Perfume Industry, in Progress in Essential Oil Research (W. D. Gruyter, ed.), Berlin, 1986.

[11] F. Speta, Flechten als Nutzpflanzen, Flechten bedrohte Wunder der Natur (W. Seipel, ed.), 1975.

[12] E. B. Bergter, Chemical Structure Immunology and Application of polysaccharides of Fungi and Lichens, Studies in Natural products Chemistry, Structure Elucidation Part B (A. Rahma, ed.), vol. 5, Elsevier, London, 1987.

[13] V. M. Dembitsky, T. Rezanka, I. A. Bychek, and M. V. Shustov, Phytochem. 31 (1992), 841.

[14] Y. Nishikawa, K. Ohki, K. Takahashi, G. Kurono, F. Fukuoka, and M. Emori, Chem. Pharm. Bull. 2211 (1974), 2692.

[15] K. O. Vartia, Ann. Med. Ex. 27 (1950), 46.

[16] R. A. Norton, Biochemical Spectroscopy, vol. 2, Adams-Hilger, Bristol, 1975.

[17] S. Kırmızıgül, Ö. Koz, H. Anıl, S. Içli, and U. Zeybek, Türk Journ. Chem., in press.

[18] M. J. S. Dewar, E. G. Zoebisch, E. F. Healy, and J. J. P. Stewart, J. Am. Chem. Soc. 107 (1985), 3902.

[19] J. P. Stewart, J. Comput. Chem. 10 (1989), 209.

[20] A. D. Bacon and M. C. Zerner, Theo. Chim. Acta. 53 (1979), 21.

[21] R. S. Mulliken, J. Chem. Phys. 23 (1995), 1833.

[22] H. Ebel (ed.), UV/VIS Atlas of Organic Compounds, VCH, 1992.

[23] A. Sharma and S. G. Schulman, Introduction to Fluorescence Spectroscopy, John Willey \& Sons, New York, 1999.

[24] D. L. Gourrierec, S. M. Ormson, and R. G. Brown, Progr. React. Kinetics 19 (1994), 211.

[25] S. Srivastava and S. K. Dogra, J. Photochem. Photobiol. A: Chem. 46 (1989), 329.

[26] T. Arai, Ethylenic Bonds, Present Status of the Photoisomerization Abut, in Advances in Photochemistry, vol. 20, Wiley \& Sons, New York, 1995.

[27] M. A. El-Sayed, J. Phys. Chem. 38 (1963), 2834.

[28] B. Valeur, Molecular fluorescence, Wiley-VCH, Weinheim (FRG), 2002.

[29] N. J. Turro, Molecular Photochemistry, Benjamin, London, 1965.

[30] L. A. Lee and R. A. Robb, J. Quantum Electronics QE-16 (1980), 777. 


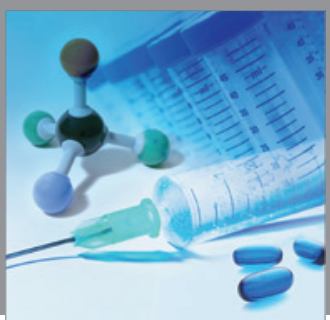

International Journal of

Medicinal Chemistry

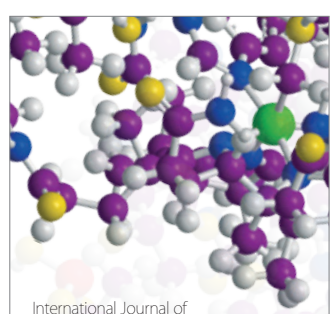

Carbohydrate Chemistry

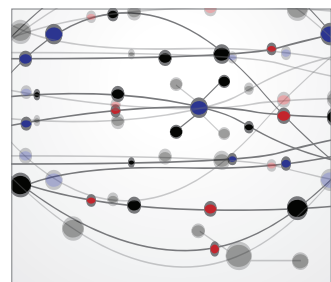

The Scientific World Journal

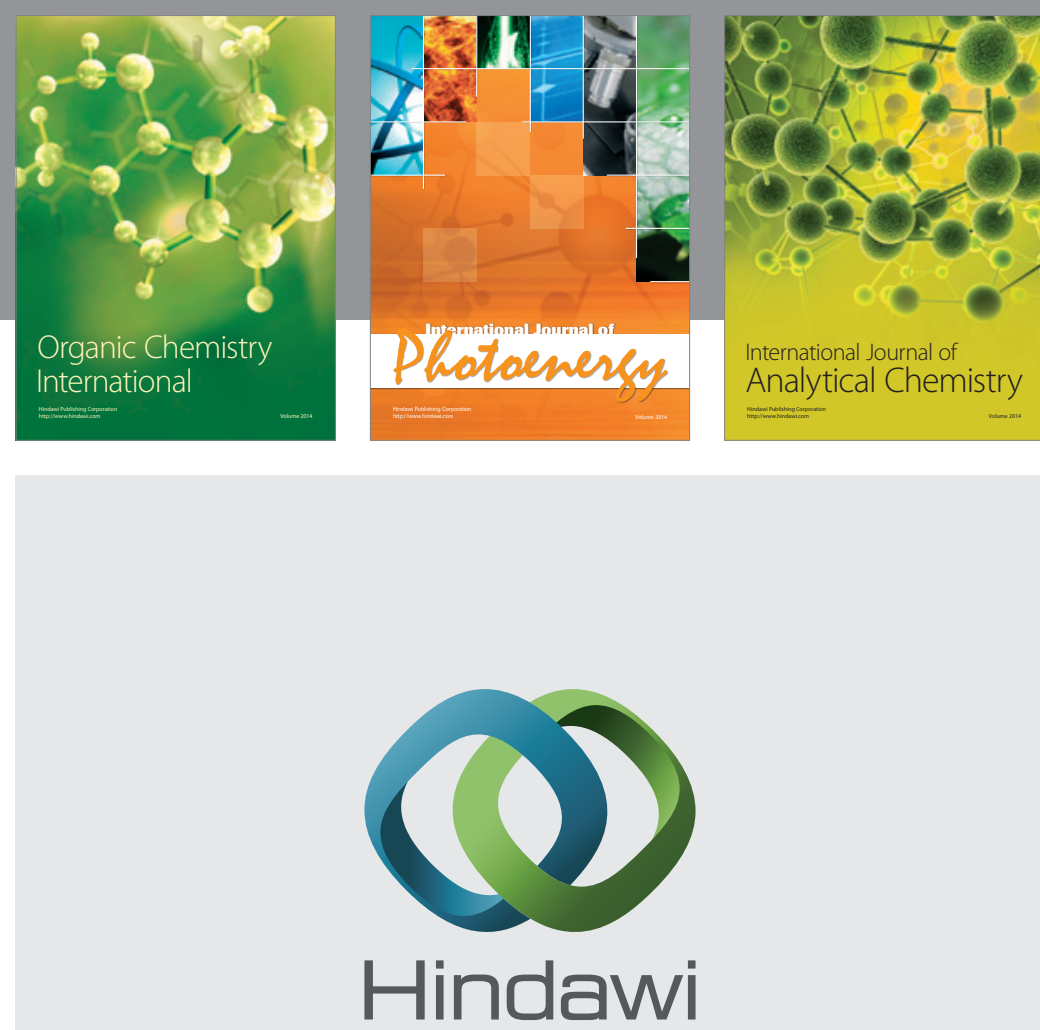

Submit your manuscripts at

http://www.hindawi.com
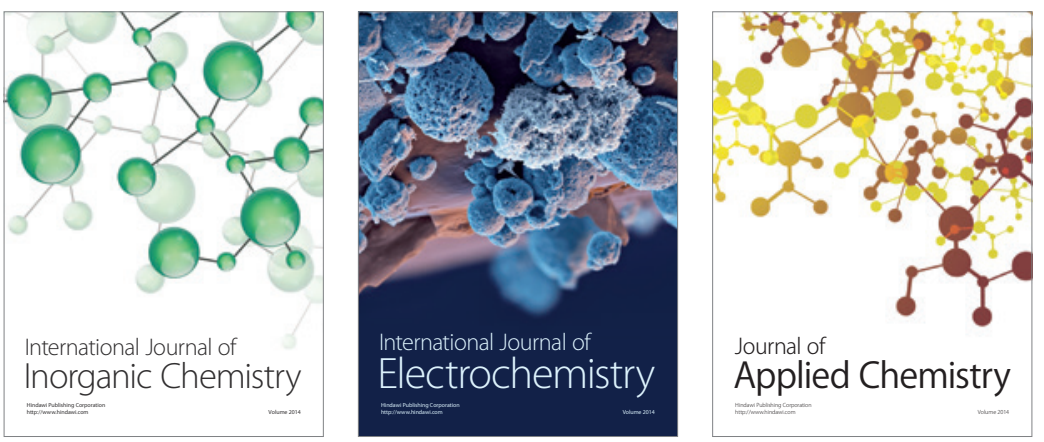

Journal of

Applied Chemistry
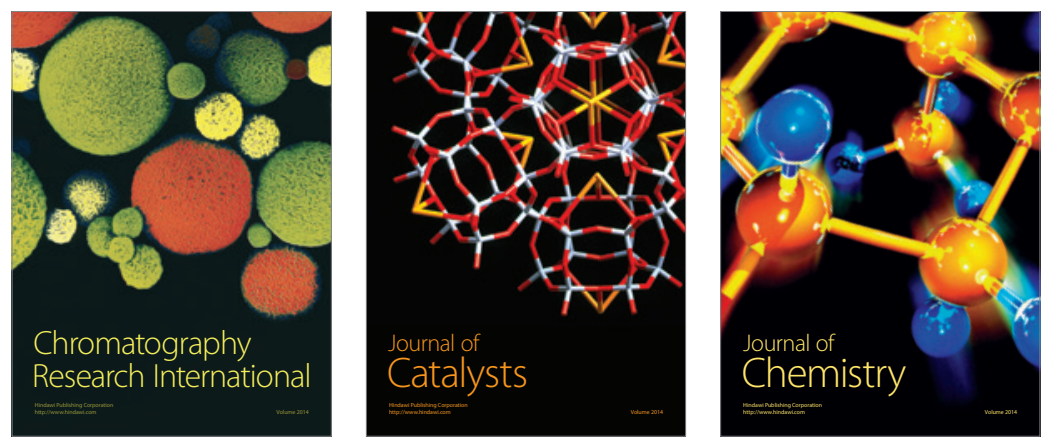
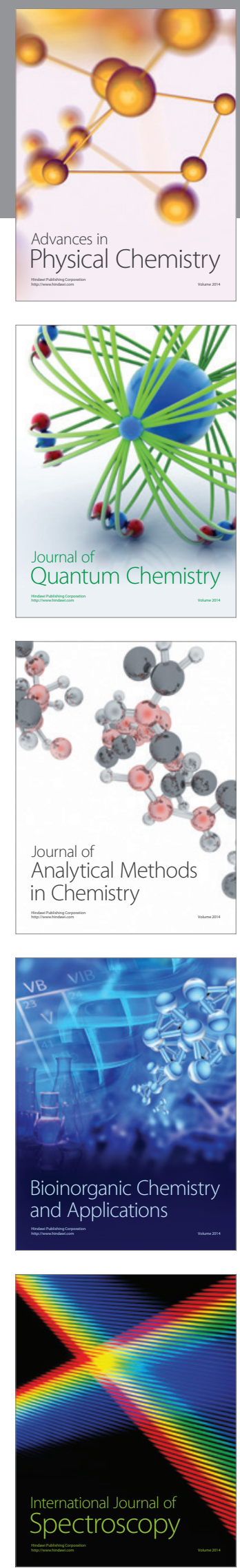